\title{
Aging-related changes in the mid-face skin elasticity in East Asian women
}

\author{
Seong Hwan Kim, \\ Seong Joo Lee, \\ Hyun Jo Kim, \\ Ju Ho Lee, \\ Hii Sun Jeong, \\ In Suck Suh \\ Department of Plastic and \\ Reconstructive Surgery, Kangnam \\ Sacred Heart Hospital, Hallym \\ University College of Medicine, Seoul, \\ Korea
}

\begin{abstract}
Background: Age-related changes in facial skin is a major concern in women. This study aimed to objectively evaluate normal skin elasticity and age-related differences in the faces of East Asian women. There are no standard values for data related to normal skin on East Asian women. Methods: We studied 129 healthy East Asian women without a history of cosmetic procedures or surgeries. Skin elasticity was assessed at the cheek and lower eyelid points, which were assessed on both the right and left sides of the face.

Results: The age of the subjects showed significant negative correlations with the R2 and R7 parameters, which represent skin elasticity after deformation.

Conclusion: We therefore concluded that the primary decrease in skin elasticity in East Asian women occurs in the midface region.
\end{abstract}

Keywords: Elasticity / Rejuvenation / Skin aging

\section{INTRODUCTION}

The overall strength and elasticity of the skin are determined by the thickness, strength, and elasticity of the epidermis, dermis, and subcutis layers. Aging-related changes in the skin layers affect the skin as a whole. The commonly noted characteristics of older skin are increased wrinkles, skin laxity, and pigmented lesions as well as decreases in skin thickness and the elastin and collagen components $[1,2]$.

Aging-related degenerative changes occur in all anatomic components such as the skeleton, adipose tissue, ligaments, and skin, including facial skin [3]. The morphologic changes in the face are most prominent after the mid-forties. The most significant changes can be found on the lower eyelid and cheeks. Wrinkles and sagging of the skin are major concerns in many

Correspondence: In Suck Suh

Department of Plastic and Reconstructive Surgery, Kangnam Sacred Heart

Hospital, Hallym University College of Medicine, 1 Singil-ro, Yeongdeungpo-gu,

Seoul 07441, Korea

E-mail: sismdps@gmail.com

Received May 7, 2019 / Revised June 10, 2019 / Accepted June 17, 2019 middle-aged women.

The face shows different aging-related changes compared to other sites of the body because it undergoes photoaging; along with the neck and forearms, the face is exposed to sunlight much more than other sites. Photoaging is characterized by severe wrinkling and pigmentation [2].

There are many studies on aging-related skin changes in Caucasians. However, to the best of our knowledge, very few studies exist researching the same in Asian populations, especially regarding aging-related changes on the face. Wrinkles are not noticeable in Asians until they are approximately in their 50s [4]. This is because of the differences in skin properties between Caucasians and Asians. We aimed to determine the aging-related changes in facial skin elasticity specifically in East Asian women.

\section{METHODS}

\section{Subjects}

A total of 129 normal Korean and Chinese women (age range, 
15-77 years) who visited our clinic from September 2015 to September 2016 were included in the study. None of the participants had any history of cosmetic surgery or other findings such as eczema, erythema, or scarring on their faces. The premenopausal subjects were evaluated when in the follicular phase of their menstrual cycle. Subjects who were pregnant or taking hormone replacement therapy were excluded. All the patients were skin type III or IV as measured using the Fitzpatrick scale.

\section{Measurements}

The subjects were assessed in comfortable conditions with the room temperature at $23^{\circ} \mathrm{C} \pm 2{ }^{\circ} \mathrm{C}$ and humidity at $35 \% \pm 5 \%$. The Cutometer (MPA 580; Courage \& Khazaka Electronic $\mathrm{GmbH}$, Cologne, Germany) was used to measure skin elasticity. The Cutometer is a device that is used to measure the mechanical and viscoelastic properties of the skin; it uses a suction mechanism. Negative pressure is created by the vacuum pump in the device, which draws the skin into the aperture of the probe. The resistance of the skin to being sucked by the negative pressure and its ability to return to its original position are measured as indicators of skin strength and elasticity [5].

The Cutometer measures the vertical deformation of the skin when it is pulled by means of a controlled vacuum into the circular aperture. The time/strain mode was used with five consecutive cycles of a 5-second suction application followed by a 3 -second relaxation period. We used a measuring probe with a 2-mm diameter and applied a constant suction of 500 mbar. Two points were measured on the right and left sides of the face each (Fig. 1). The first is a point on the lower eyelid $1.5 \mathrm{~cm}$ below the mid-pupillary line. The second is the point where the vertical line from the lateral canthus and the horizontal line from the oral commissure meet (Fig. 2). Point 1 represents the right lower eyelid, 2 the left lower eyelid, 3 the right cheek, and 4 the left cheek.

In each of the measured regions, the Ua (the difference between the maximum deformation of the first vacuum period and the deformation after 1 second of normal pressure), the Ue (immediate distension of the skin within the first 0.1 second of the first vacuum period), the Uv (the difference between the deformation after 0.1 second and the maximal deformation of the first vacuum period), the Uf (the final distension at the end of the first vacuum period), and the Ur (the immediate relaxation within the first 0.1 second after the end of the first vacuum period) were obtained from the skin deformation curves (Fig. 3). The curves of the obtained skin deformation values

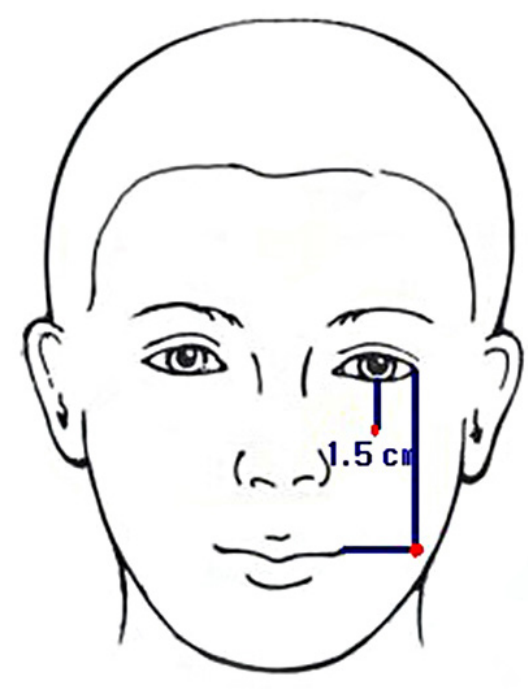

Fig. 2. The measuring points on the lower eyelid and cheek. Each point was measured on both sides of the face (red points).
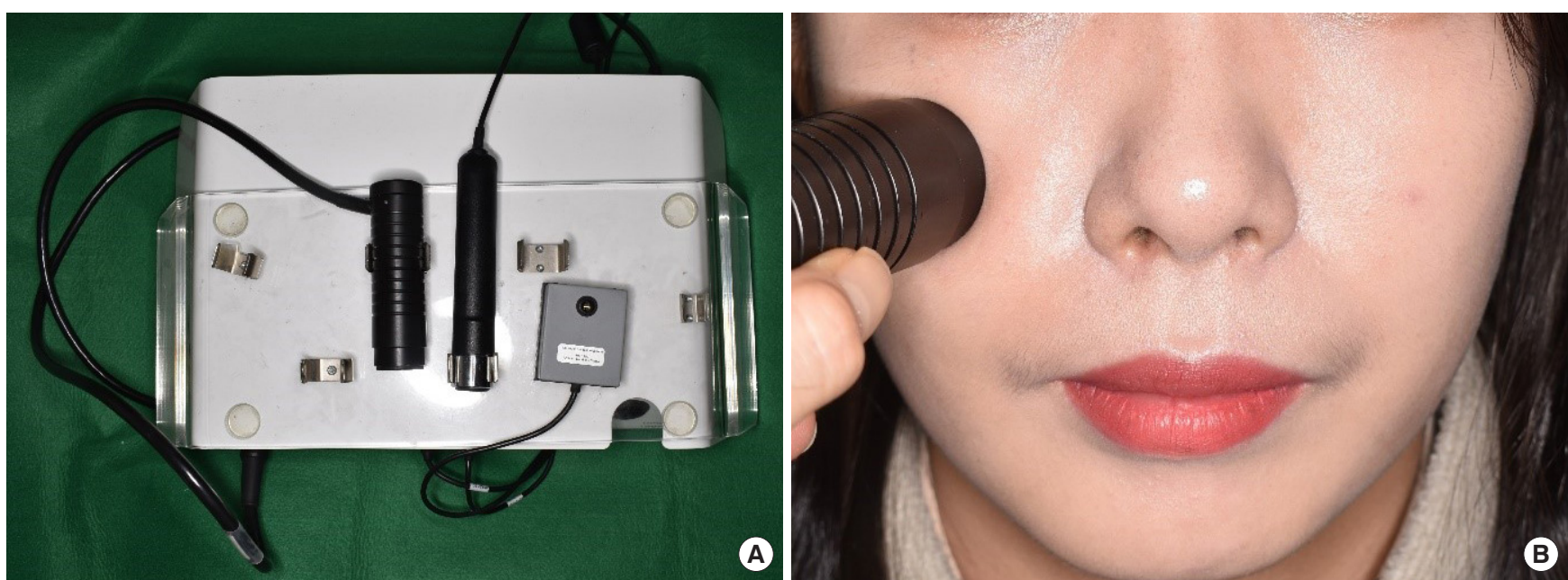

Fig. 1. (A) Cutometer device is shown. (B) Skin elasticity is measured on both lower eyelid and cheek with a probe. 


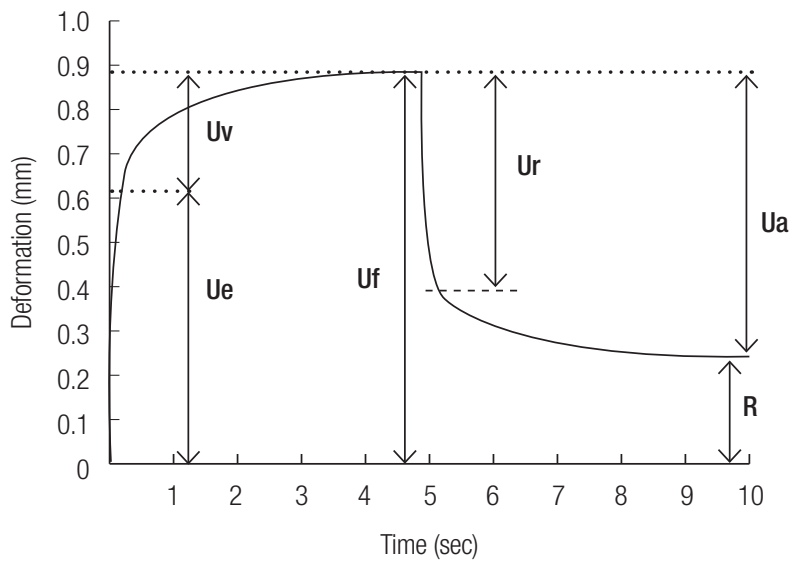

Fig. 3. Skin deformation curve obtained using the Cutometer. Uf, final distension at the end of the first vacuum period; Ua, difference between the maximum deformation of the first vacuum period and the deformation after 1 second of normal pressure; Ur, immediate relaxation within the first 0.1 second after the end of the first vacuum period; Ue, immediate distension of the skin within the first $0.1 \mathrm{sec}-$ ond of the first vacuum period; Uv, difference between the deformation after 0.1 second and the maximal deformation of the first vacuum period; $\mathrm{R}$, residual deformation after the release of the first suction.

Table 1. Parameters used for evaluating the skin properties

\begin{tabular}{|c|c|c|}
\hline Parameter & Representation & Equivalent \\
\hline Ro & The final distension of the first curve & Uf \\
\hline R1 & The ability to return to the original state & Uf-Ua \\
\hline $\mathrm{R} 2$ & $\begin{array}{l}\text { The overall elasticity of the skin, including creep and } \\
\text { creep recovery }\end{array}$ & $\mathrm{Ua} / \mathrm{Uf}$ \\
\hline R3 & The last maximum highest point of the last curve & Last Uf \\
\hline R4 & The last minimal lowest point of the last curve & Last R \\
\hline R5 & The net elasticity & Ur/Ue \\
\hline R6 & $\begin{array}{l}\text { The ratio of viscoelastic to elastic extension, also called } \\
\text { the viscoelastic ratio }\end{array}$ & Uv/Ue \\
\hline $\mathrm{R} 7$ & The ratio of elastic recovery to the total deformation & Ur/Uf \\
\hline R8 & The Ua of the first curve & First Ua \\
\hline R9 & $\begin{array}{l}\text { The residual deformation at the end of the measuring } \\
\text { cycle }\end{array}$ & R3-R0 \\
\hline
\end{tabular}

Uf, final distension at the end of the first vacuum period; Ua, difference between the maximum deformation of the first vacuum period and the deformation after $1 \mathrm{sec}-$ ond of normal pressure; Ur, immediate relaxation within the first 0.1 second after the end of the first vacuum period; Ue, immediate distension of the skin within the first 0.1 second of the first vacuum period; Uv, difference between the deformation after 0.1 second and the maximal deformation of the first vacuum period.

were analyzed using the embedded software, and the R0 to R9 parameters could be deducted from the U parameters (Table 1).

\section{Statistical analysis}

The parameters R0 to R9 on each area (locations 1 to 4 ) and their relationships to age were analyzed using Pearson correlation coefficient. All statistical analyses were conducted using SPSS version 22.0 (IBM Corp., Armonk, NY, USA).
Table 2. Overview of correlation coefficients of parameter and location

\begin{tabular}{lccccccc}
\hline & \multicolumn{5}{c}{ Location } \\
\cline { 2 - 3 } Parameter & \multicolumn{2}{c}{ Lower eyelid } & & \multicolumn{2}{c}{ Cheek } & \multirow{2}{*}{ Mean } \\
\cline { 2 - 3 } & Right & Left & & Right & Left & \\
\hline R0 & -0.032 & -0.077 & & 0.000 & -0.107 & -0.053 \\
R1 & 0.144 & -0.008 & & $0.341^{\text {a) }}$ & $0.247^{\text {a) }}$ & 0.090 \\
R2 & $-0.551^{\text {b) }}$ & $-0.521^{\text {b) }}$ & & $-0.419^{\text {b) }}$ & $-0.444^{\text {b) }}$ & $-0.472^{\text {b) }}$ \\
R3 & -0.017 & -0.091 & & 0.029 & -0.117 & -0.048 \\
R4 & $0.206^{\text {a) }}$ & 0.171 & & $0.400^{\text {b) }}$ & $0.249^{\text {a) }}$ & $0.252^{\text {b) }}$ \\
R5 & -0.255 & -0.087 & & -0.289 & -0.104 & $-0.121^{\text {b) }}$ \\
R6 & -0.123 & 0.059 & & 0.014 & 0.118 & 0.012 \\
R7 & $-0.318^{\text {b) }}$ & $-0.548^{\text {b) }}$ & & $-0.605^{\text {b) }}$ & $-0.636^{\text {b) }}$ & $-0.523^{\text {b) }}$ \\
R8 & -0.076 & -0.110 & & -0.088 & -0.173 & $-0.109^{\text {a) }}$ \\
R9 & 0.066 & -0.143 & & $0.223^{\text {a) }}$ & -0.103 & 0.003 \\
\hline
\end{tabular}

Pearson correlation coefficient of the parameter with age of the patients. R2 and R7 are highly correlated with aging of the skin.

${ }^{\text {a) }} p<0.05 ;{ }^{\text {b) }} p<0.01$

\section{RESULTS}

The mean age of the patients was $49.4 \pm 16.9$ years. The Spearman coefficients of each parameter related with age are shown in Table 2. Among the relative parameters, R2 (Ua/Uf) and R7 (Ur/Uf) showed high correlations. The correlation coefficient of R2 for locations $1,2,3$, and 4 was $-0.551,-0.521,-0.419$, and -0.444 , respectively. The correlation coefficient of R7 for locations 1 to 4 was $-0.318,-0.548,-0.605$, and -0.636 , respectively. The mean correlation coefficient of R2 and R7 with aging was -0.472 and -0.523 , respectively. The scatter plots of R2 and R7 are shown in Figs. 4 and 5. Both graphs showed the negative correlation of the parameters with age. All the data were statistically significant $(p<0.01)$. The mean variations of the parameter R2 and R7 are shown in Tables 3 and 4 . The subjects were divided into groups according to their ages, with a 10-year interval as one group. A gradual decrease of the average on each location was seen.

Other relative parameters are $\mathrm{R} 5(\mathrm{Ur} / \mathrm{Ue}$,$) and R6 (Uv/Ue),$ which correspond to net elasticity and viscoelasticity ratio, respectively. As shown in Table 2, there were no statistically significant correlations of the parameters R5 and R6 with age.

Among the absolute parameters, R1 (Uf-Ua) and R4 (the last minimal lowest point of the last curve) showed a low correlation with age. The R1 parameter was 0.341 and 0.247 on the right and left cheek, respectively. The R4 parameter on locations 1 to 4 was $0.206,0.171,0.400$, and 0.249 , respectively. All the data except R4 on left lower eyelid were statistically significant $(p<0.05)$.

The other parameters R0 (Uf), R3 (the last maximum highest 




Fig. 4. Scatter plots of R2 according to age.

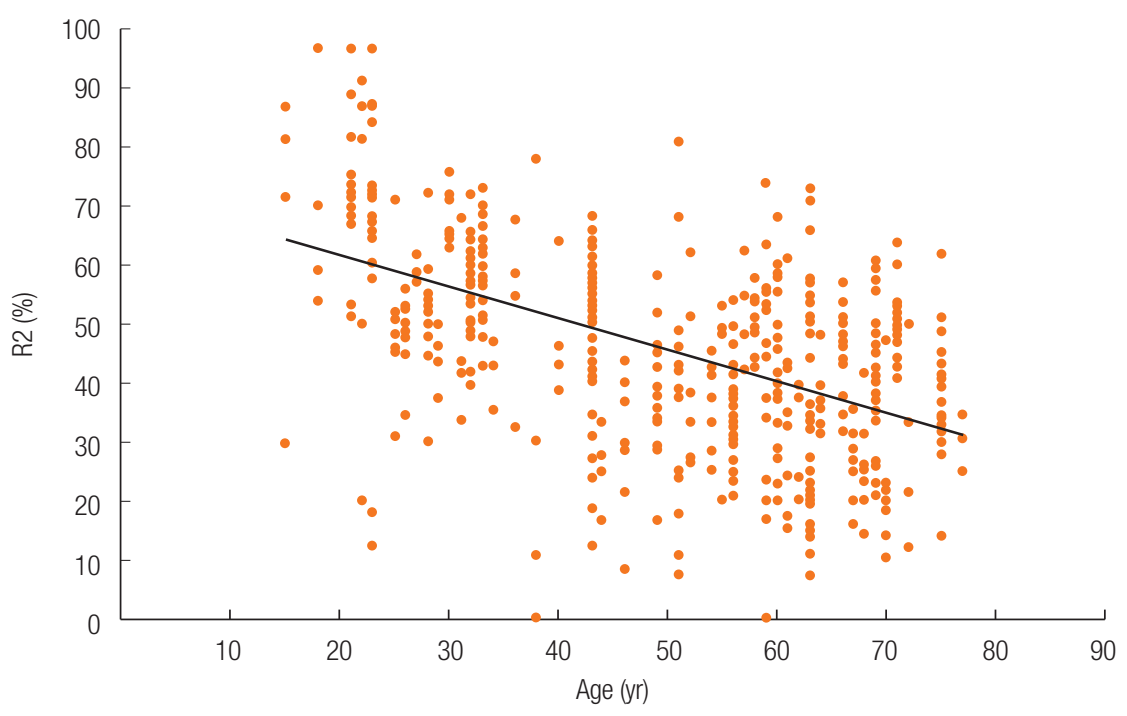

Fig. 5. Scatter plots of R7 according to age.

Table 3. Age-related variations in the mean parameter $\mathrm{R} 2$ on each location

\begin{tabular}{|c|c|c|c|c|c|}
\hline \multirow{3}{*}{$\begin{array}{l}\text { Age group } \\
\text { (yr) }\end{array}$} & \multirow{3}{*}{$\begin{array}{l}\text { No. in } \\
\text { group }\end{array}$} & \multicolumn{4}{|c|}{ Parameter $\mathrm{R} 2$, mean $\pm \mathrm{SD}$} \\
\hline & & \multicolumn{2}{|c|}{ Lower eyelid } & \multicolumn{2}{|c|}{ Cheek } \\
\hline & & Right & Left & Right & Left \\
\hline $15-30$ & 24 & $0.87 \pm 0.05$ & $0.86 \pm 0.08$ & $0.90 \pm 0.05$ & $0.86 \pm 0.08$ \\
\hline $31-40$ & 16 & $0.80 \pm 0.06$ & $0.79 \pm 0.10$ & $0.71 \pm 0.14$ & $0.84 \pm 0.12$ \\
\hline $41-50$ & 19 & $0.74 \pm 0.10$ & $0.75 \pm 0.14$ & $0.73 \pm 0.19$ & $0.79 \pm 0.13$ \\
\hline $51-60$ & 29 & $0.69 \pm 0.17$ & $0.69 \pm 0.15$ & $0.70 \pm 0.24$ & $0.76 \pm 0.16$ \\
\hline $61-77$ & 41 & $0.63 \pm 0.16$ & $0.65 \pm 0.16$ & $0.62 \pm 0.21$ & $0.66 \pm 0.20$ \\
\hline Average & 129 & $0.73 \pm 0.16$ & $0.73 \pm 01.6$ & $0.72 \pm 0.21$ & $0.76 \pm 0.17$ \\
\hline
\end{tabular}

point of the last curve), and R9 (R3-R0) represent the changes after repeated suction test of the Cutometer with R1 and R4 [6].
Table 4. Age-related variations in the mean parameter R7 on each location

\begin{tabular}{|c|c|c|c|c|c|}
\hline \multirow{3}{*}{$\begin{array}{l}\text { Age group } \\
\text { (yr) }\end{array}$} & \multirow{3}{*}{$\begin{array}{l}\text { No. in } \\
\text { group }\end{array}$} & \multicolumn{4}{|c|}{ Parameter $\mathrm{R} 7$, mean $\pm \mathrm{SD}$} \\
\hline & & \multicolumn{2}{|c|}{ Lower eyelid } & \multicolumn{2}{|c|}{ Cheek } \\
\hline & & Right & Left & Right & Left \\
\hline $15-30$ & 24 & $0.51 \pm 0.17$ & $0.70 \pm 0.20$ & $0.63 \pm 0.16$ & $0.66 \pm 0.12$ \\
\hline $31-40$ & 16 & $0.48 \pm 0.14$ & $0.52 \pm 0.17$ & $0.59 \pm 0.09$ & $0.55 \pm 0.13$ \\
\hline $41-50$ & 19 & $0.44 \pm 0.17$ & $0.40 \pm 0.12$ & $0.47 \pm 0.14$ & $0.47 \pm 0.14$ \\
\hline $51-60$ & 29 & $0.34 \pm 0.15$ & $0.47 \pm 0.16$ & $0.40 \pm 0.12$ & $0.38 \pm 0.10$ \\
\hline $61-77$ & 41 & $0.38 \pm 0.13$ & $0.38 \pm 0.16$ & $0.37 \pm 0.14$ & $0.38 \pm 0.13$ \\
\hline Average & 129 & $0.42 \pm 0.16$ & $0.48 \pm 0.20$ & $0.47 \pm 0.17$ & $0.47 \pm 0.16$ \\
\hline
\end{tabular}

The parameters R0, R3, and R9 showed no significant correlation with age. 


\section{DISCUSSION}

The skin on every part of the body changes with aging. As the face is the most prominent part and is visible first, many women are concerned about signs of aging on the face, considering an aesthetic perspective. Sagging of the skin on the mid-face is mostly visible on the lower eyelids and cheeks. Therefore, we chose to assess the skin of the lower eyelid and cheek to represent facial aging most accurately.

Various intrinsic and extrinsic factors, such as age, gender, temperature, humidity and hormonal changes and affect the skin's properties. Controlling all the variants influencing the elasticity of the skin is impossible. On our study, we controlled the variants of gender, location, temperature, humidity and affection of menstruation. However other variants like time of measurement, hydration status and seasonal changes were not controlled on our study. Tightly controlled study can reveal accurate results where many variants can affect the dependent variable of the study.

The limitation of this study is small number of patients and limited area of measurement. Large numbers of data are needed for statistical analysis and precise average of normal skin on each age. Further study with large number of patients is needed. The patients were from South Korea and China. We think the data can represent the East Asian women, but not the whole Asian women. More studies of skin elasticity using Cutometer with different races can show the differences of skin quality between different races such as Caucasians, Asians and Blacks, etc.

R2 (Ua/Uf) represents the ratio of total recovery of skin on normal pressure after the deformation and R7 (Ur/Uf) represent the immediate recovery of skin on normal pressure after deformation. Therefore, R2 and R7 represent the elasticity of the skin and its ability to recover its original position. Our results showed a negative correlation of these parameters with aging, suggesting that the ability of the skin to recover from deformation decreases with aging. Nishimura and Tuji [7] and Cua et al. [8] reported that a decrease in R7 is significantly correlated with facial aging. The skin's elastin fiber network is responsible for the R7 parameter. R6 ( $\mathrm{Uv} / \mathrm{Ue})$ is known to increase with age $[7,8]$, but the increase in R6 was not significant in this study.

R3, R4, and R9 are correlated with skin fatigue. Repeated suction and release with the Cutometer provoked skin fatigue, resulting in decreased elasticity and increased maintenance of the deformed position [6]. Dobrev [6] reported that adult skin shows a higher degree of fatigue than young skin. Parameters R3, R4, and R9 positively correlated with age in our study. This shows that skin in older subjects has reduced capability to restore its initial position. Multiple suction with the Cutometer increases the R3 and R4 and decreases R9, which is more significant in the older participants. In our study, the significant changes were found only in R4 and even that had a low Spearman coefficient. We think this was because we used only five repeated suctions for one measurement and with increased repetition, the R3, R4, and R9 changes may become increasingly significant in older patients.

Takema et al. [9] reported that absolute parameters like Ue, $\mathrm{Uv}, \mathrm{Ur}$, and Uf tend to increase with age in the forearm but decreased in the face. They explained the reason was that high exposure to sunlight reduced the extensibility and elastic recovery of facial skin. Other studies have mentioned that degeneration and thickening of elastin fibers occurs in the skin exposed to sunlight $[10,11]$. In our study, the absolute parameters like R0, $\mathrm{R} 1$, and R8 seemed to be unrelated to aging. We think this is because the facial skin is different compared to the skin on the other sites that are more frequently exposed to the sunlight.

There are many studies on aging-related changes in the skin. On the microscopic study, the aged skin shows reduced the thickness, disorganization of the elastin fibers decreased the number of the collagen bundles [12-14]. Microscopic changes in the skin components and the thickness are considered to be the cause of decreased skin elasticity. Skin on the abdomen and forearm in women also showed decreased elasticity in other studies $[7,8,15]$. The elasticity of the mid-face in East Asian women seems to match that of the other body parts.

Laser therapy such as intense pulsed light and ablative fractional laser is effective in reducing wrinkles and improving skin elasticity in Chinese women [14]. The lasers tighten the skin by penetrating into the deep dermis and stimulating fibroblast proliferation and collagen rearrangement [16]. Facial aesthetic surgeries such as face lifting and lower blepharoplasty can improve the skin elasticity by pulling the skin mechanically. Recently, the ultrasonic waves, which are effective and non-invasive, have been actively used for facial rejuvenation [17]. Other methods like injections and massages can also be used to improve the facial skin elasticity and reduce the wrinkles. There are many methods to improve the age-related changes of skin elasticity. However, the effectiveness of these methods needs to be measured in a quantitative manner, by measuring the changes they bring about in the skin. The knowledge of normal values of elasticity of the mid-face in East Asian women can be used to evaluate the quantitative effectiveness of each treatments. Further studies can be done using these data to find out the quantitative improvements of facial rejuvenating surgeries or procedures.

There are many devices to measure skin elasticity, such as the Cutometer, Dermaflex, DermaLab, and Tonometry. Among them, the Cutometer has been proved to be the most effective 
to evaluate the mechanical properties and viscoelasticity of the skin [18]. We therefore used the Cutometer in our study to measure skin properties in East Asian women and determine the mean values for the parameters.

In conclusion, the properties of facial skin are different from those of other body parts, especially with regards to the effects of frequent exposure to sunlight. We used the Cutometer to evaluate skin elasticity changes in East Asian women and found that the midface region is the primary area where skin elasticity reduces with age.

\section{NOTES}

\section{Conflict of interest}

No potential conflict of interest relevant to this article was reported.

\section{Ethical approval}

The study was performed in accordance with the principles of the Declaration of Helsinki. Written informed consents were obtained.

\section{Patient consent}

The patient provided written informed consent for the publication and the use of her image.

\section{ORCID}

Seong Hwan Kim https://orcid.org/0000-0001-6831-5621

Hii Sun Jeong https://orcid.org/0000-0001-9408-8207

In Suck Suh https://orcid.org/0000-0002-9793-4031

\section{REFERENCES}

1. Sumino H, Ichikawa S, Abe M, Endo Y, Ishikawa O, Kurabayashi $\mathrm{M}$. Effects of aging, menopause, and hormone replacement therapy on forearm skin elasticity in women. J Am Geriatr Soc 2004;52:945-9.

2. Chung JH. Photoaging in Asians. Photodermatol Photoimmunol Photomed 2003;19:109-21.

3. Wulc AE, Sharma P, Czyz CN. The anatomic basis of midfacial aging. In: Hartstein ME, Wulc AE, Holck DE, editors. Midfacial rejuvenation. New York: Springer; 2012. p. 15-8.
4. Goh SH. The treatment of visible signs of senescence: the Asian experience. Br J Dermatol 1990;122 Suppl 35:105-9.

5. Dobrev HP. A study of human skin mechanical properties by means of Cutometer. Folia Med (Plovdiv) 2002;44:5-10.

6. Dobrev H. Application of Cutometer area parameters for the study of human skin fatigue. Skin Res Technol 2005;11:120-2.

7. Nishimura M, Tuji T. Measurement of skin elasticity with a new suction device: relation to age, anatomical region, sun-exposure and comparison with diseased skin. Jpn J Dermatol 1992;122: 1111-7.

8. Cua AB, Wilhelm KP, Maibach HI. Elastic properties of human skin: relation to age, sex, and anatomical region. Arch Dermatol Res 1990;282:283-8.

9. Takema Y, Yorimoto Y, Kawai M, Imokawa G. Age-related changes in the elastic properties and thickness of human facial skin. Br J Dermatol 1994;131:641-8.

10. Kligman AM. Early destructive effect of sunlight on human skin. JAMA 1969;210:2377-80.

11. Lavker RM. Structural alterations in exposed and unexposed aged skin. J Invest Dermatol 1979;73:59-66.

12. Braverman IM, Fonferko E. Studies in cutaneous aging: I. the elastic fiber network. J Invest Dermatol 1982;78:434-43.

13. Lovell CR, Smolenski KA, Duance VC, Light ND, Young S, Dyson M. Type I and III collagen content and fibre distribution in normal human skin during ageing. Br J Dermatol 1987;117: 419-28.

14. Mei XL, Wang L. Ablative fractional carbon dioxide laser combined with intense pulsed light for the treatment of photoaging skin in Chinese population: a split-face study. Medicine (Baltimore) 2018;97:e9494.

15. Castelo-Branco C, Pons F, Gratacos E, Fortuny A, Vanrell JA, Gonzalez-Merlo J. Relationship between skin collagen and bone changes during aging. Maturitas 1994;18:199-206.

16. Li YH, Chen JZ, Wei HC, Zhang L, Xu HH, Xu TH, et al. A Chinese experience of fractional ultrapulsed $\mathrm{CO} 2$ laser for skin rejuvenation. J Cosmet Laser Ther 2010;12:250-5.

17. Wulkan AJ, Fabi SG, Green JB. Microfocused ultrasound for facial photorejuvenation: a review. Facial Plast Surg 2016;32:26975.

18. Verhaegen PD, van der Wal MB, Middelkoop E, van Zuijlen PP. Objective scar assessment tools: a clinimetric appraisal. Plast Reconstr Surg 2011;127:1561-70. 